\title{
S. Gianfausto, C. De Michele, N.T. Kottegoda, R. Renzo: Extremes in Nature: An Approach Using Copulas
}

\author{
Springer, 2007
}

\author{
Jaume Masoliver
}

Received: 3 December 2008 / Accepted: 10 December 2008 / Published online: 19 December 2008 (C) Springer Science+Business Media, LLC 2008

This monograph deals with both theoretical and practical aspects of the mathematical theory of extremes. Extreme events in nature continue to cause uncountable casualties and economic losses. Therefore, an acquaintance with the statistical distribution of extreme events is an essential first step in assessing the effectiveness of any program of disaster management in responding to extreme events caused by nature.

The theory of extremes was initiated in the late nineteen twenties by the works of Frechet, Fisher, and Tippet and subsequently developed by Gnedenko and Gumbel later in the forties and early fifties. Originally it dealt with univariate distributions and the central result from this period is the Frechet-Tippet theorem which states that under suitable conditions the asymptotic distributions of extremes are restricted to be of three types (Gumbel, Frechet and Weibull). The authors review this result and devote the first chapter to the one-dimensional theory. The results are presented in a clear and rigorous way although with few proofs (there is an extended bibliography at the end of the book where one can find the desired proofs). Nevertheless, the emphasis of the book is on applications mostly to geophysical problems.

In the second chapter the authors address the mathematical theory of multivariate extremes where one studies the joint occurrence of critical values. This is a relative modern topic in which analogues of the univariate distribution are thoroughly studied. In the remaining three chapters of the book the authors apply the theory of copulas - a rather modern development in mathematical statistics which studies joint distributions with fixed marginalsto the analysis of multivariate extremes. As before, propositions are clearly stated without proof but many geophysical applications are discussed.

This is a concise and short book of a bit over 280 pages with five chapters and three appendices dealing with the simulation of copulas, dependence and families of copulas. The book surely will be interesting and useful to researchers and practitioners in the areas of geophysics and environmental sciences and engineering. It can be also useful to any one interested in having a rigorous summary of the main results and modern developments of the theory of extreme events in the context of physical applications.

J. Masoliver ( $\bowtie)$

Departament de Fisica Fonamental, Universitat de Barcelona, Barcelona, Spain

e-mail: Jaume.masoliver@ub.edu 